EPJ Web of Conferences 59, 04007 (2013)

DOI: $10.1051 /$ epjconf/20135904007

(C) Owned by the authors, published by EDP Sciences, 2013

\title{
Dynamic stabilization of Rayleigh-Taylor instability in ablation fronts
}

\author{
A.R. Piriz ${ }^{1, a}$, L. Di Lucchio ${ }^{1, b}$, G. Rodriguez Prieto ${ }^{1}$ and N.A. Tahir ${ }^{2}$ \\ ${ }^{1}$ E.T.S.I. Industriales, Universidad de Castilla-La Mancha and Instituto de Investigaciones \\ Energéticas, 13071 Ciudad Real, Spain \\ 2 GSI Helmholtzzentrum für Schwerionenforschung Darmstadt, Planckstrasse 1, \\ 64291 Darmstadt, Germany
}

\begin{abstract}
Dynamic stabilization of Rayleigh-Taylor instability in an ablation front is studied by considering the simplest possible modulations in the acceleration. Explicit analytical expressions for the instability growth rate and for the boundaries of the stability region are obtained by considering a sequence of Dirac deltas. Besides, general square waves allow for studying the effect of the driving asymmetries on the stability region as well as the optimization process. The essential role of compressibility is phenomenologically addressed in order to find the constraints it imposes on the stability region.
\end{abstract}

\section{INTRODUCTION}

In inertial confinement fusion (ICF) the control of Rayleigh-Taylor instability (RTI) during the implosion has the potential for reducing the driver energy. Dynamic stabilization (DS) driven by the vertical vibration of the front has been proposed for reducing the instability growth rate and the minimum perturbation wave number $k=2 \pi / \lambda$ ( $\lambda$ is the perturbation wavelength) that is stable $[1,2]$. Previous works have assumed that a sinusoidal vibration would be the most tractable analytically. Instead, it leads to a Mathieu equation that makes the finding of the stability regions a very difficult task.

Here, we present a study for the particular case of an ablation front directly driven by ion beams for which DS can be of special interest [3-5]. However, application of the methods presented here to ablation fronts driven by thermal diffusion are straightforward. First, we consider a modulation in the driving acceleration consisting in a sequence of Dirac deltas. Then, we study different types of modulations involving general squares waves which should be more representative of experimentally accessible drivings. This type of waveform has also the advantage that allows for studying asymmetries in the acceleration amplitudes to get insight on the optimization of DS of ablative RTI.

\section{BASIC EQUATIONS AND DISPERSION RELATION}

We consider the RTI instability of an ablation front in the framework of the sharp boundary model as in Refs. [2, 3, 6-8]. The interface is placed at $y=\xi(x, t)$ and separates two fluids of densities $\rho_{2}$ and $\rho_{1}<\rho_{2}$, ahead and behind the front, respectively. The gravitational field $\mathbf{G}(t)$ is pointing in the

\footnotetext{
ae-mail: roberto.piriz@uclm.es

${ }^{\mathrm{b}}$ Permanent address: INFN Bologna and Physics Department, University of Bologna, Italy
}

This is an Open Access article distributed under the terms of the Creative Commons Attribution License 2.0, which permits unrestricted use, distribution, and reproduction in any medium, provided the original work is properly cited. 


\section{EPJ Web of Conferences}

positive $y$-axis direction which is taken in the direction opposite to the density gradient. The information regarding the density profiles at both sides of the front is incorporated by considering the self-consistent density jump $r_{D}=\rho_{1} / \rho_{2}$, with $\rho_{1}$ and $\rho_{2}$ taken as the densities at a distance $k^{-1}$ of the interface. Thus, we can get the equation of motion of the interface due to the instability $[3,6,8]$ :

$$
\ddot{\xi}+\frac{4 k v_{2}}{1+r_{D}} \dot{\xi}+\left[\phi_{0} \frac{k^{2} v_{2}^{2}}{r_{D}}-A_{T} k G(t)\right] \xi=0,
$$

where $A_{T}=\left(1-r_{D}\right) /\left(1+r_{D}\right)$ is the Atwood number, $v_{2}$ is the ablation velocity, and $\phi_{0}$ is the fraction of the energy that is transported up to the front by thermal conduction [3]. The density jump $r_{D}$ is given by the mechanism of energy transport and, by taking $r_{D} \ll 1$, we can write $r_{D} \approx\left(n k L_{2}\right)^{n}$, where $L_{2}$ is the characteristic length of the density/temperature gradient in the corona region $(y>0)$ close to the front. We will take $n=1 / 3$ without loosing generality in the conclusions. On the other hand, $G(t)=g+b \Gamma(\omega t)$ and $b=\omega^{2} A$, where $g$ is the background acceleration, and $\Gamma(\omega t)$ is a periodic function that oscillates with frequency $\omega$ and amplitude $A$. We will assume first that the oscillatory acceleration is of the form: $\Gamma(\omega t)=\delta(\omega t-2 m \pi)-\delta[\omega t-(2 m+1) \pi]$, where $\delta(\tau)$ are Dirac deltas and $m$ is an integer. To solve Eq. (1) it is convenient to introduce the dimensionless variables $\tau=\omega t$ and $x=\xi / \xi_{0}$, where $\xi_{0}$ is the initial perturbation amplitude. Thus, Eq. (1) reads:

$$
\begin{gathered}
\ddot{x}+2 D \dot{x}+\left[K^{2}-\beta \Gamma(\tau)\right] x=0, \\
\kappa=\frac{k v_{2}^{2}}{g} ; \varpi=\frac{\omega v_{2}}{g} ; \\
D=\frac{2 \kappa}{\varpi} ; \beta=\frac{\kappa}{\varpi^{2}} \frac{b}{g} ; K^{2}=\frac{\kappa}{\varpi^{2}}\left[\left(\frac{\kappa}{\kappa_{c}}\right)^{2 / 3}-1\right] ; \kappa_{c}=\frac{r_{D}\left(\kappa_{c}\right)}{\phi_{0}} .
\end{gathered}
$$

In Eq. (3) we have introduced the dimensionless cut-off wave number $\kappa_{c}$ which is the only parameter required to characterize the RTI in the non-oscillating ablation front $(b=0)$. As it can be appreciated, $\kappa_{c}$ is determined by the Froude number $F r_{2}=v_{2}^{2} / g L_{2}$ and by the fraction $\phi_{0}$ of the energy flux that is transported by thermal diffusion and it becomes larger as smaller is the value of $\phi_{0}$. Eq. (2) is a damped Hill' equation and according to the Floquet theory it has solutions of the following general form: $x(\tau)=P(\tau) e^{\sigma \tau}$; where $\sigma=\gamma / \omega$ and $P(\tau)$ is a periodic function and $\gamma$ is the instability growth rate. In addition, it yields the dispersion relation that gives the dimensionless growth rate $\sigma$ and, for $\sigma=0$, determines the upper ("+") and the lower ("-") limits of marginal stability:

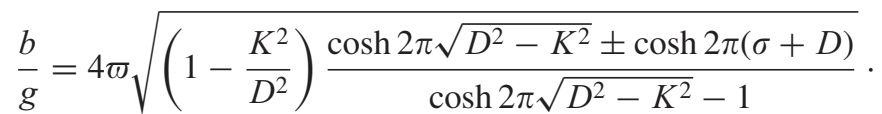

Typical limits of marginal stability are shown in Fig. 1 i). The upper limit has a minimum which determines the maximum value $b / g)_{\max }$ that can be used for DS which will be referred as the upper boundary of stability. The lower limit determines the minimum value $b / g)_{\min }$ that is required in order to dynamically stabilize all the wave numbers $\kappa \geq \kappa_{m}$, with $\kappa_{m}<\kappa_{c}$, and it will be referred as the lower boundary of stability. In order to have a region of stability in the parameter space of the problem it is necessary that $\left.b / g)_{\min }<b / g\right)_{\max }$. In this manner we can construct the stability charts of $b / g$ as a function of $\varpi$ that give the stability region for a given values of the parameter $\kappa_{c}$ and of the minimum wave number $\kappa_{m}$ that we want to stabilize. Typical chart are shown in Fig. 1 ii) for $\kappa_{c}=0.3$. This figure shows that the smaller the minimum value $\kappa_{m}$ of the wave numbers that we want to stabilize for a given value of the cut-off wavenumber $\kappa_{c}$, the larger the relative amplitude $b / g$ and the larger frequency $\varpi$ of the modulation are necessary for stabilization. In the same manner, it results that the smaller $\kappa_{c}$, the more difficulty to reduce $\kappa_{m}$ to a given fraction of $\kappa_{c}$, thus requiring higher values of $b / g$ and $\varpi$. 

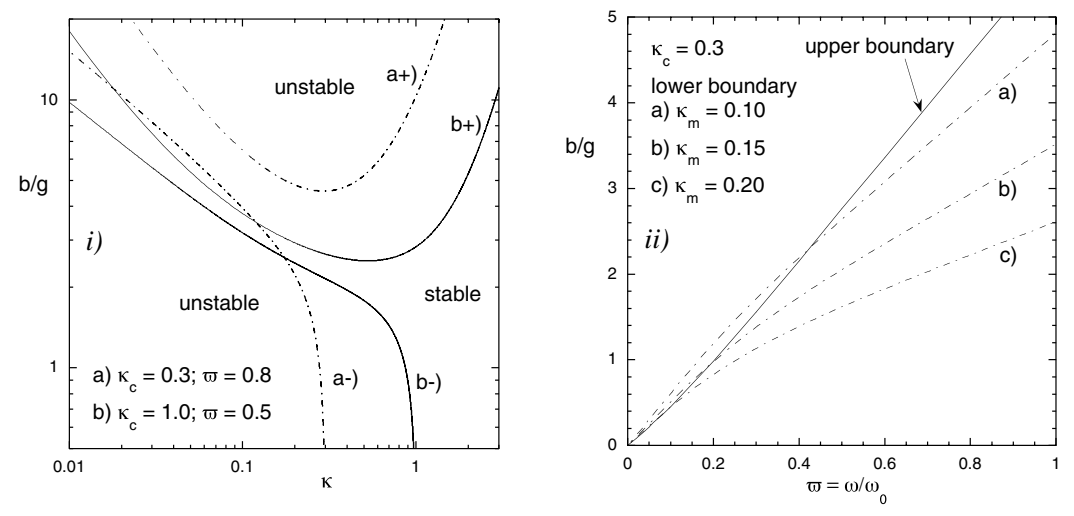

Figure 1. i) Dimensionless accelerations $b / g$ for marginal stability as functions of the dimensionless wave number $\kappa$ for $\kappa_{c}=0.3, \varpi=0.8$ and for $\kappa_{c}=1, \varpi=0.5$. Symbols "+" and "-" denote the stability upper and lower limits, respectively. ii) Stability region for $\kappa_{c}=0.3$. The lower boundary is given for $\kappa_{m}=0.10,0.15$, and 0.20 .

Figure 1 ii) shows that relatively large values of $b / g$ are required in order to be inside the stability region. It would be impossible to generate such large oscillating accelerations if the unablated material ahead the ablation front were perfectly incompressible. Actually, DS in an ablation front can work because the layers of the unablated material close to the ablation surface are compressed and decompressed by the oscillating pressure [1,2]. Thus, it generates local accelerations $b$ that can be considerably larger than the background acceleration $g$ affecting the whole mass of the accelerated shell. The ablation pressure by itself can push the ablation front but is unable to pull it. Thus, this latter part of the oscillating cycle depends on the decompression of the layer ahead the front. The process for which values of $b / g>1$ can be achieved in an ablation front as well as an estimation of the region thickness affected by the vibration is analyzed here in a phenomenological manner in order to understand what new constraints are imposed by the compressibility effects on the stability region.

For this, we notice that the minimum wave number $k_{m}$ above which the front is dynamically stabilized cannot be arbitrary chosen since it will depend on the oscillation frequency [2]. In fact, the thickness of the shell region that will be affected by the oscillation is the one that can be reached by the sound waves, traveling with velocity $c_{s 2}$, during an oscillation characteristic time of the order of $\omega^{-1}$. That is $k_{m}^{-1} \approx \Delta y \approx c_{s 2} \omega^{-1}$. Using the dimensionless variables defined in Eq. (3), we get: $\kappa_{m} \approx M_{2} \varpi$; where $M_{2}=v_{2} / c_{s 2}$ is the Mach number in the relatively dense and cold material ahead the ablation front $(y<0)$. This equation shows that the larger is the frequency $\varpi$, the larger will be the minimum value of the of $\kappa_{m}$, suggesting that too large frequencies may not stabilize sufficiently small wave numbers.

On the other hand, large frequencies are necessary to produce large local accelerations $b$. In fact, the amplitude $b$ of the acceleration modulation, produced by an oscillation of amplitude $\Delta p$ in the ablation pressure can be estimated as $b \approx \Delta p / \rho_{2} \Delta y$ [2]. Since the ablation pressure is $p \approx \rho_{2} g d$, where $d$ is the shell thickness, and introducing dimensionless variables, we get: $b / g \approx(\Delta p / p)\left(\varpi / M_{2}\right)$. Taking into account that $\Delta p / p<1$, it turns out that relatively high frequencies $\left(\varpi \gg M_{2}\right)$ are required to produce values of $b / g$ considerably larger than unity. So, we should use the lowest possible frequency that allows for entering in the stability region. The consequences of the two previous conditions on the boundaries of the stability region can be seen by introducing the frequency dependent value of $\kappa_{m}$ given above into the negative branch of Eq. (4), with $\sigma=0$, in order to calculate the lower limit for a fixed value of the Mach number $M_{2}$. Once this is done, the region of the stability chart that can actually be achieved will be determined by the minimum between the $b / g$ values given above and the positive branch of Eq. (4). A typical case is shown in Fig. 2 i) for a reference case with $\kappa_{c}=0.3$. One can see that if the Mach number of the dense shell is known, a unique value of the minimum wave number $\kappa_{m}$ that can 

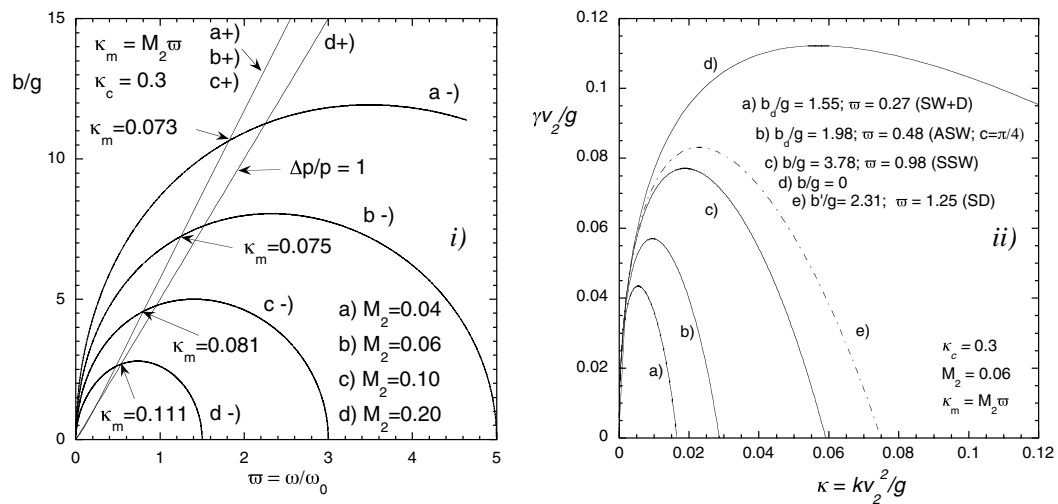

Figure 2. i) Stability region for $\kappa_{c}=0.3$. The lower boundaries (“-”) are given for $\kappa_{m}=M_{2} \varpi$, and different values of the Mach number $M_{2}=0.04,0.06,0.10$, and 0.20 . The upper boundaries ("+") correspond to the incompressible case of Fig. 1 ii) for the smallest values of $M_{2}$, and to a pressure modulation of a $100 \%$ for the case $M_{2}=0.20$. ii) Instability growth rates produced by using different types of drivings: a) negative square wave+positive Dirac deltas (SW+D); b) asymmetric square wave (ASW with $c=\pi / 4)$; c) symmetric square wave (SSW); d) no driving $(b=0)$; e) symmetric Dirac deltas $(\mathrm{SD})$.

be stabilized is determined, as well as the value of $b / g$ and of the oscillation frequency $\varpi$ that must be used. For instance, if $M_{2}=0.1$ we will be able to stabilize the wave numbers larger than $\kappa_{m} \approx 0.08$ and for this it will be necessary to achieve an acceleration modulation $b / g \approx 4.54$ and a frequency $\varpi \approx 0.8$. For a sufficiently small Mach number $M_{2}$, depending of the value of $\Delta p / p$ in the ablation pressure modulation, the previous expression for $b / g$ does not impose any new constraint to the upper boundary. In any case, the minimum value of $\kappa_{m}$ is rather insensitive to the exact value of $M_{2}$ but, instead, it considerably affects the required values of $b / g$ and $\varpi$.

\section{EFFECT OF THE VIBRATION WAVEFORM}

The minimum value $k_{m}$ of the perturbation wavenumber, for a given value of the cut-off wavenumber $k_{c}$, that is dynamically stabilized can be used for comparing the performance of the different types of drivings. In Fig. 2 ii) we have represented the resulting growth rates for the best situations corresponding to each waveform together with the reference case with no front vibration $(b=0)$. We find that although the considerable $50 \%$ reduction of the dynamic cut-off wave number $\kappa_{m}$ between the symmetric Dirac deltas and the SSW cases [Fig. 2 ii c) and e)], it is not really a great relative improvement in comparison with the already achieved from the reference case with a cut-off wave number $\kappa_{c}=0.3$ [Fig. 2 ii d)]. This indicates that the best performance of DS is not strongly dependent of the particular modulation waveform provided that it is perfectly symmetric. In particular, we should expect similar results for the sinusoidal driving traditionally considered in literature. However, when asymmetries are considered, a stronger reduction of the growth rate and of the cut-off wavenumber is obtained [Fig. 2 ii b) and a), respectively]. In particular, asymmetries with a short duration and large positive acceleration followed by long duration and small negative acceleration, perform better than the opposite case. The best performance happens for the limiting case when the positive acceleration is a Dirac delta.

In conclusion, for a typical target [4], we have $k_{c} \approx 750 \mathrm{~cm}^{-1}\left(l_{c}=k_{c} r_{0} \approx 150\right.$, where $r_{0} \approx 0.2 \mathrm{~cm}$ is the target radius), with $g \approx 10^{16} \mathrm{~cm} / \mathrm{s}^{2}$ and $v_{2} \approx 2 \times 10^{6} \mathrm{~cm} / \mathrm{s}$, so that $\kappa_{c} \approx 0.3$. Therefore, we can get a one order of magnitude reduction in the cut-off wave number by using a pressure modulation with $\left.\omega \approx 2.5 \times 10^{9} \mathrm{~s}^{-1}, \Delta p / p\right)_{d} \approx 0.25$, and $\left.\Delta p / p\right)_{d} \approx 1.75$ [ø $\approx 0.5$, and $b_{d} / g \approx 2$ in Fig. 2 ii b) $]$, which are values well into the present experimental capabilities. 
IFSA 2011

This work has been supported by the MICINN of Spain (ENE2009-09276).

\section{References}

[1] J. P. Boris, Comments Plasma Phys. Controlled Fusion 3, 1 (1977)

[2] R. Betti, R. L. McCrory, and C. P. Verdon, Phys. Rev. Lett. 71, 3131 (1993)

[3] S. A. Piriz, A. R. Piriz, and N. Tahir, Phys. Plasmas 16, 082706 (2009)

[4] B. G. Logan, L. J. Perkins, and J. J. Barnard, Phys. Plasmas 15, 072701 (2008)

[5] A. R. Piriz, Plasma Phys. Controlled Fusion 28, 387 (1986); 28, 1167 (1986)

[6] R. Betti et al., Phys. Plasmas 2, 3844 (1995); 3, 2122 (1996)

[7] J. G. Wouchuk and A. R. Piriz, Phys. Plasmas 2, 493 (1995)

[8] A. R. Piriz, J. Sanz, and L. F. Ibañez, Phys. Plasmas 4, 1117 (1997) 